\title{
Positive effects of rehabilitation training with the L-EXOS in chronic stroke
}

\author{
Antonio Frisoli ${ }^{*}$, Edoardo Sotgiu ${ }^{*}$, Caterina Procopio ${ }^{* * \dagger}$, Carmelo Chisari $^{* \dagger}$, Bruno Rossi ${ }^{* \dagger}$, \\ Massimo Bergamasco* \\ (*)PERCRO Laboratory, TECIP, Scuola Superiore Sant'Anna, Italy \\ $(*+)$ Nerurorehabilitation Unit, Neuroscience Dept., University of Pisa, Italy \\ E-mail: a.frisoli@sssup.it (contact author)
}

\begin{abstract}
This paper presents the results of the evaluation training performed in a group of chronic stroke patients with L-Exos device. The effects of training were assessed both by means of clinical evaluation in terms of Fugl-Meyer and Modified Ashworth assessment scales, and of functional evaluation, by means of Bimanual Activity Scale. Moreover at each session an automatic assessment of performance was made through the robot, and this was compared with the outcome of functional evaluation. Interestingly we found a significant improvement of both clinical and functional evaluation, and that the automatic assessment performed by the robot in terms of movement smoothness represents a strong predictor of transfer of functional ability to activity of every day life.
\end{abstract}

\section{Introduction}

Rehabilitation training in stroke performed with robot assistance can lead to significant motor recovery when the patient is actively involved with volitional effort in the exercise, and this is proposed in a task-oriented ecological environment.

A class of rehabilitation robots that deserve a particular interest in upper limb rehabilitation is constituted by active exoskeletons. Exoskeleton are robots with kinematic isomorphic to the human arm, that can be worn on the user arm. They present several significant advantages compared to end-effector based system [1]. They may track the full arm kinematics, so not only the end-effector position, typically the hand, but the full kinematic chain of the arm, and consequently apply a force assist at the level of each joint, e.g. shoulder, elbow and wrist. The workspace covered by these systems is three dimensional and large, so that the training can be conducted on fully spatial movements and along different directions.
The Light-Exoskeleton [1] is a right-arm rehabilitation robot used for stroke patients rehabilitation; it is composed of five degree-offreedom (DoF), of which four active, i.e. shoulder and elbow flexion/extension, shoulder internal/external rotation and, abduction/adduction (Figure 2).

The distinguishing feature of an exoskeleton based device is that it can provide both a passive and active assistance to the patient during the execution of the task.

In the simplest way the assistance provided by the robot can consist in a gravity counterbalancing of weight of the arm, as this has been proved to enlarge the workspace of the arm [2] and to be effective for motor recovery.

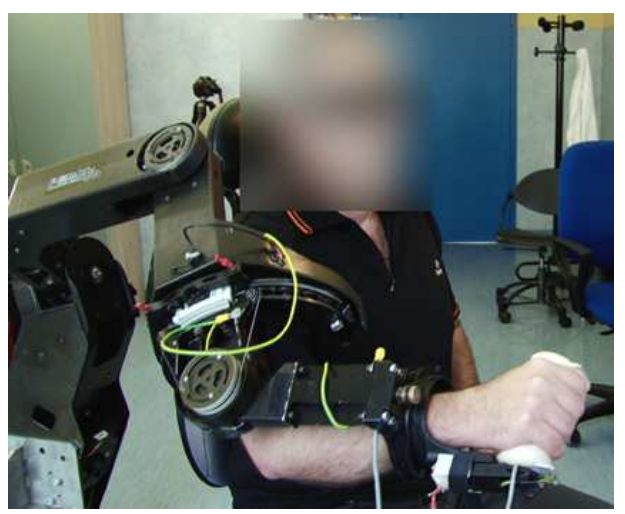

Figure 1 The L-Exos system worn by a patient

Alternatively the robot can provide a guided assistance to the task to be performed, according to an impedance-based model or more advanced controls [3]. In this abstract we present the results of a rehabilitation training performed on a group of chronic stroke patients, who underwent a rehabilitation training and assessment performed.

This is an Open Access article distributed under the terms of the Creative Commons Attribution-Noncommercial License 3.0, which permits unrestricted use, distribution, and reproduction in any noncommercial medium, provided the original work is properly cited. 


\section{Methods}

Patients were administered a robotic training with the L-Exos system with training scenarios projected on a stereoscopic projection wall.

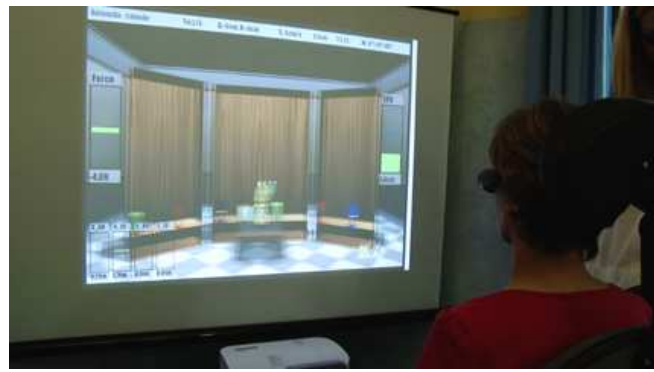

Figure 2 The rehabilitation system with the gaming scenario

The rehabilitation training consisted in two training exercises and one evaluation test, performed with the assistance of a robotic device.

The first exercise consisted in a reaching task performed with an impedance assistance by the robot with level of assistance modulated by the robot according to the position error (impedance assistance) or with variable gain adjusted according to the force input by the patient (triggered assistance), to enhance patient's active participation in the task.

The second training exercise consisted in a training scenario in which the patient has to compose a virtual puzzle with the gravity assistance only provided by the robot. The exercise was then followed by an evaluation session allowing to assess the performance in movement execution over different direction of space, since a set of target to be reached where placed in the vertical plane, along twelve possible positions arranged equally spaced along a circumference (Figure 4).
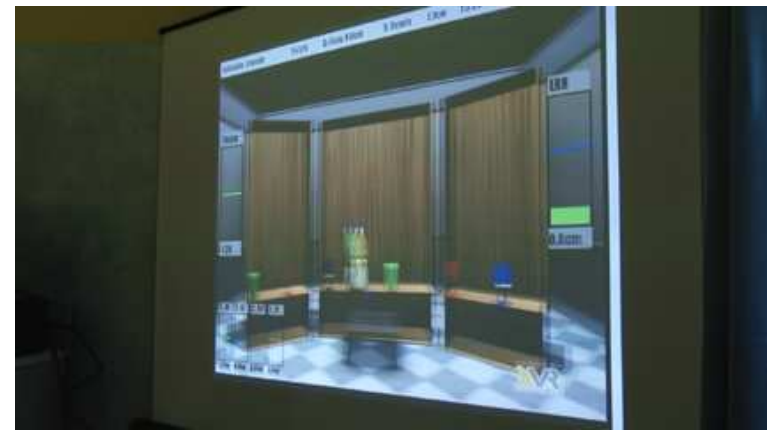

Figure 3 The reaching training scenario

Both smoothness and time of execution of movement were measured as indexes of performance through the robot in this evaluation session. Movement time was computed as the time to move from the start position in the centre to the target, while smoothness index was computed by counting the number of peaks in the velocity profile of movement

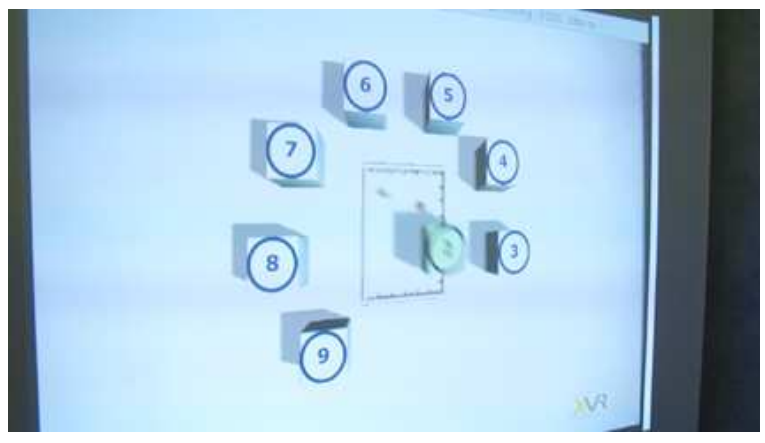

Figure 4 The evaluation session

\section{Participants}

Seven patients with stroke event occurred at least six months ahead ( 2 females, 5 males, $60.14 \pm 12.35$ years old) were enrolled in the treatment.

At the enrollment and discharge of treatment patients underwent clinical evaluation performed with upper limb Fugl-Meyer Assessment Score (FMA, 66 pts) and Modified Ashworth Scale (MA, on 19 muscle groups), plus a functional assessment performed with a standardized test, the Bimanual Activity test, consisting in evaluating in terms of time and quality of movement (estimated on 0-4 scale) the execution by the patient of a set of ADL tasks requiring bimanual coordination.

At the enrollment the average FMA assessment score was of $25.5 \pm 12.99$ and the average MA of $11.88 \pm 9.96$.

Three out of seven patients underwent also a follow-up clinical assessment after 10 months since the end of treatment to evaluate the retention of regained function.

\section{Results}

We observed a significant clinical improvement in all patients. Clinical assessment at the end of treatment revealed an increase of FMA to $31.43 \pm 15.41(\mathrm{p}<0.02)$, with no significant change in spasticty (MA after treatment $10.57 \pm 7.52$ ).

We observed on the functional level a significant reduction of time in the execution of bimanual movement from $17.19 \pm 3.83$ to $12.33 \pm 5.38 \mathrm{sec}$ $(\mathrm{p}<0.02)$ with an overall increase of quality of movement from $1.99 \pm 0.77$ to $2.79 \pm 0.92(\mathrm{p}<0.03)$.

The assessment of the two indexes of performance performed with the robot revealed a similar trend, with a marked decrease of movement time from $2.57 \pm 1.17$ 
to $1 \pm 0.68 \mathrm{sec}(\mathrm{p}<0.004)$ and marked increase of smoothness going from $6.9 \pm 1.19$ to $2.51 \pm 1.8$ $(p<0.003)$, where decrease of the smoothness index means increase of smoothness of movement.

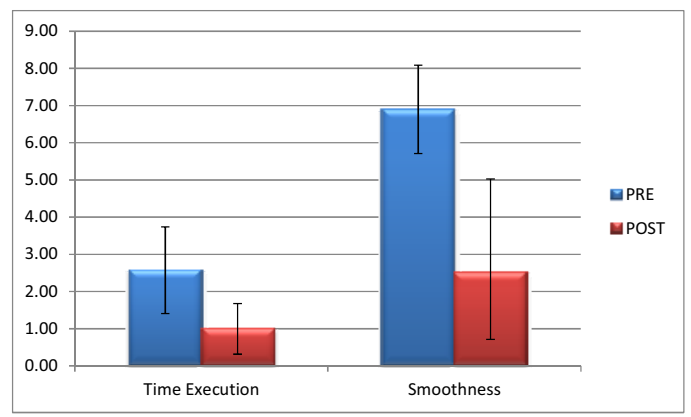

Figure 5 Assessment of movement performance with robot

We investigated the correlation of the functional indexes (Bimanual Activity Scale) with the robot assessment scale, to assess whether the improvement of performance observed with the robot was effectively transferred as ability to perform ADL activity

We found a strong correlation between the smoothness index and the time and quality of movement assessed with the Bimanual Activity Scale, as shown in Figure 6 and Table 1.

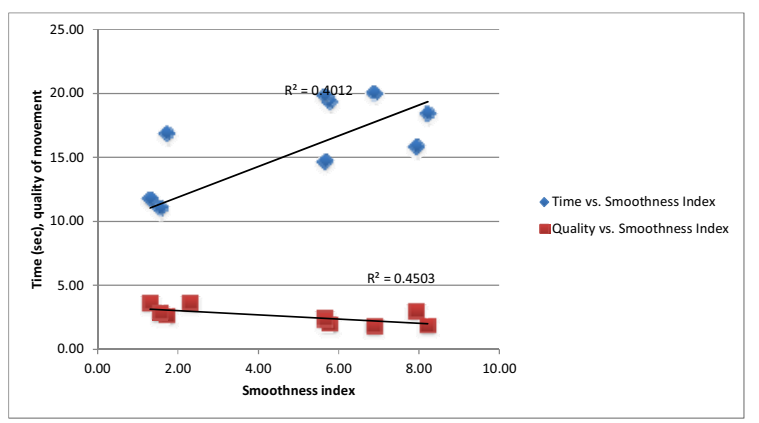

Figure 6 Correlation of bimanual activity index with smoothness of movement

Moreover the performance improvement in movement execution was analyzed in terms of different directions of space by means of the robot-based assessment, by means of radial plots, shown in Figure 8 and Figure 9. It is possible to see from the picture how a marked and significant improvement is obtained mainly in the movements executed to reach targets in the ipsilateral space, while no significant improvement is obtained in the contralateral space.

\begin{tabular}{|l|l|r|r|}
\hline \multicolumn{2}{|l|}{} & ExecutionTime & Smoothness \\
\hline BimanualTime & $\begin{array}{l}\text { Pearson } \\
\text { Correlation }\end{array}$ & .489 & $.633\left(^{*}\right)$ \\
\hline & $\begin{array}{l}\text { Sig. (2- } \\
\text { tailed) }\end{array}$ & .151 & .049 \\
\hline & $N$ & 10 & 10 \\
\hline BimanualQuality & $\begin{array}{l}\text { Pearson } \\
\text { Correlation }\end{array}$ & -.616 & $-.671\left(^{*}\right)$ \\
\hline & $\begin{array}{l}\text { Sig. (2- } \\
\text { tailed) }\end{array}$ & .058 & .034 \\
\hline & $N$ & 10 & 10 \\
\hline
\end{tabular}

Table 1 Correlation indexes of Bimanual Activity Scale with robot-based assessment

Moreover in the small subgroup of patients that underwent a follow-up evaluation at month 10 , we found a percentage improvement of FMA of $5.97 \%$ that was maintained also at the follow-up assessment, were still the $5.59 \%$, as shown in Figure 7.

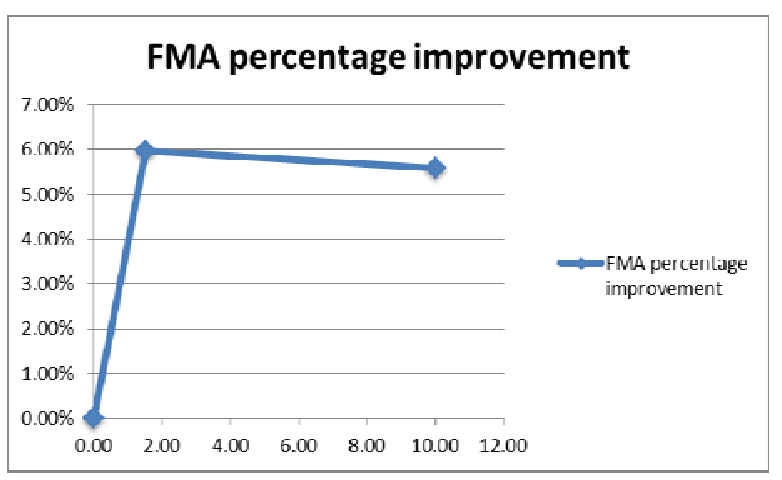

Figure 7 Follow-up evaluation

\section{Discussion and conclusions}

Confirming our previous results, we have found that the rehabilitation training might lead to different improvement of performance in the contralateral and ipsilateral space.

Moreover we have found that the improvement in smoothness of movement represents a predictive indicator of both clinical assessment performed with clinical scale and also of regained ability to perform activity of every day life. This represents an important result, since it allows to directly map the measured performance to improvements into functional activity. 


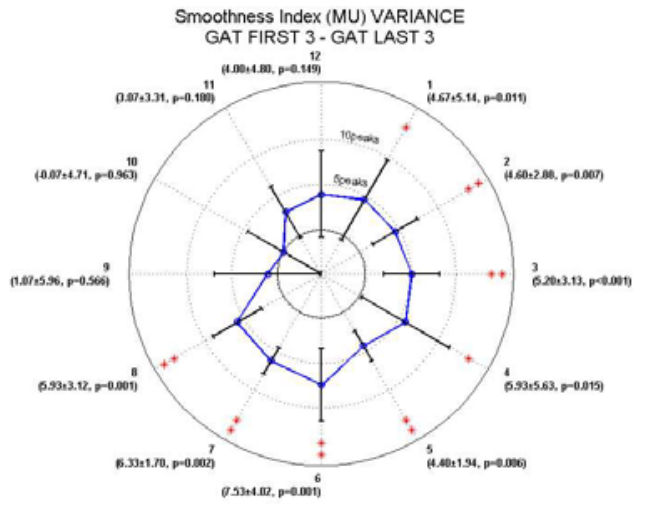

Figure 8 Percentage improvement of smoothness index, ${ }^{* *} \mathbf{p}<0.01, * \mathbf{p}<0.05$

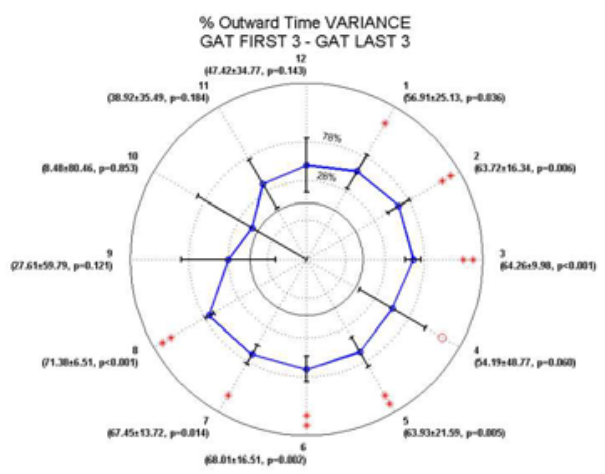

Figure 9 Improvement of time execution, $* * \mathbf{p}<0.01$, $* \mathbf{p}<0.05$
The first preliminary results from follow-up evaluation performed at 10 months of distance since treatment, allowed us also to hypothesize that such a regain of function is preserved over time.

\section{References}

[1] Frisoli, A., F. Salsedo, M. Bergamasco, B. Rossi, and M.C. Carboncini, A force-feedback exoskeleton for upper-limb rehabilitation in virtual reality. Applied Bionics and Biomechanics, 2009. 6(2): p. 115-126.

[2] Sukal, T.M., M.D. Ellis, and J.P.A. Dewald, Shoulder abduction-induced reductions in reaching work area following hemiparetic stroke: neuroscientific implications. Experimental Brain Research, 2007. 183(2): p. 215-223.

[3] Frisoli, A., L. Borelli, A. Montagner, S. Marcheschi, C. Procopio, F. Salsedo, M. Bergamasco, M.C. Carboncini, and B. Rossi. Robot-mediated arm rehabilitation in Virtual Environments for chronic stroke patients: a clinical study. in IEEE ICRA, International Conference on Robotics \& Automation. 2008: IEEE. 